\title{
LEARNING DISABILITIES AND NF-1; MRI ABNORMALITIES
}

The significance of abnormalities on MRI was evaluated in 40 children with NF-1, aged 8 to 16 years, at the Children's Hospital, Sydney, Australia. The distribution of Full Scale IQ scores was bimodal, one group with and a second group without cognitive impairment. Children with areas of increased signal intensity on MRI (unidentified bright objects, UBO+), accounting for $62.5 \%$ of the study population, had lower IQs than those without these lesions. Language function, visuomotor integration, academic achievement, and coordination were also impaired in the UBO+ group. IQ and achievement in the UBO- group were similar to the general population. (North $\mathrm{K}$ et al. Specific learning disability in children with neurofibromatosis type 1: Significance of MRI abnormalities. Neurology May 1994;44:878-883). (Reprints: Dr Kathryn North, Genetics Division, Fegan 10, Children's Hospital, 300 Longwood Ave, Boston, MA 02115).

COMMENT. UBOs in children with neurofibromatosis-1, representing areas of developmental dysplasia and aberrant myelination, are associated with cognitive deficits. Patients identified with UBOs should be managed separately from the UBO- group in determining the need for special education services.

Similar findings have been reported from the Johns Hopkins Hospital, Baltimore, MA (Hofman KJ, Denckla MB et al. Neurofibromatosis type 1: The cognitive phenotype. I Pediatr April 1994;124:S1-8). In a study of 12 families, children with NF-1 compared to unaffected siblings had a lower Full Scale IQ multifocal cognitive deficits, reading disability, and neuromotor deficit. Paired cognitive differences correlated with the number of brain lesions on MRI.

\section{INFANTILE SPASMS AND NF-1}

Two patients, ages 7 and 2 years, with neurofibromatosis type 1 complicated by infantile spasms are reported from the Pediatric Institutes, Siena and Catania, Italy. ACTH and anticonvulsants were ineffective. Both children were mentally retarded and one had UBOs on MRI. The authors believe that the association of NF-1 and infantile spasms is not a coincidence and infantile spasms should be included among the clinical manifestations of NF-1. (Fois A, Tine A, Pavone L. Infantile spasms in patients with neurofibromatosis type 1 . Child's Nerv Syst 1994;10:176-179). (Respond: Dr A Fois, Pediatric Institute, University of Siena, I-53100 Siena, Italy).

COMMENT. The authors cite 7 additional cases of infantile spasms with NF-1 in the literature, the first reference to this association originating from Japan (Kurokawa T et al. Pediatrics 1980;65:81). They include a report by Motte et al of 15 cases, all responsive to steroids and having a good mental outcome (1992, unpublished data). A reference to 2 cases reported in a population study of 135 patients with NF-1 in Wales was omitted (Huson SM et al. In: Progress in Pediatric Neurology I, 1991, Chicago, PNB Publ, p 372). Infantile spasms and hypsarrhythmia occur much less frequently with NF-1 than in tuberous sclerosis, but the association is more prevalent than expected. 